Cinémas

Revue d'études cinématographiques

Journal of Film Studies

\title{
Terminator 2. Technologie et identité sexuelle
}

\section{Sylvestre Meininger}

Volume 5, numéro 3, printemps 1995

Cinélekta 1

URI : https://id.erudit.org/iderudit/1001151ar

DOI : https://doi.org/10.7202/1001151ar

Aller au sommaire du numéro

Éditeur(s)

Cinémas

ISSN

1181-6945 (imprimé)

1705-6500 (numérique)

Découvrir la revue

Citer cet article

Meininger, S. (1995). Terminator 2. Technologie et identité sexuelle. Cinémas, 5(3), 131-150. https://doi.org/10.7202/1001151ar

\section{Résumé de l'article}

À travers l'analyse de la représentation des deux machines créées par le film Terminator 2 (Cameron, 1992), ce travail se propose d'exposer et de comprendre le rapport entre technologie et masculinité dans le cinéma hollywoodien. Ce rapport particulier prenant ses origines au plus profond de la culture occidentale, nous nous appuierons sur l'étude d'un des produits les plus extrêmes de cette culture : le fascisme en Allemagne entre les deux guerres. La perception fasciste des corps, masculin et féminin nous permettra en effet de comprendre les angoisses et les désirs exprimés par la forme des deux machines antagonistes dans Terminator 2. Parallèlement, la comparaison, diégétique cette fois, de ce film avec le premier épisode de la serie, The Terminator (Cameron, 1984), nous aidera à évaluer de quelle manière ces représentations de machines influent sur le contenu narratif de Terminator 2. 


\title{
Terminator 2. Technologie et identité sexuelle
}

\section{Sylvestre Meininger}

\author{
RÉSUME
}

A travers l'analyse de la représentation des deux machines créées par le film Terminator 2 (Cameron, 1992), ce travail se propose d'exposer et de comprendre le rapport entre technologie et masculinité dans le cinéma hollywoodien. Ce rapport particulier prenant ses origines au plus profond de la culture occidentale, nous nous appuierons sur l'étude d'un des produits les plus extrêmes de cette culture: le fascisme en Allemagne entre les deux guerres. La perception fasciste des corps, masculin et féminin nous permettra en effet de comprendre les angoisses et les désirs exprimés par la forme des deux machines antagonistes dans Terminator 2. Parallèlement, la comparaison, diégétique cette fois, de ce film avec le premier épisode de la série, The Terminator (Cameron, 1984), nous aidera à évaluer de quelle manière ces représentations de machines influent sur le contenu narratif de Terminator 2.

\section{ABSTRACT}

By examining the representation of two machines created in the film Terminator 2 (Cameron, 1992), this study seeks to uncover and understand the relationship between technology and masculinity in Hollywood cinema. Since the origin of this relationship lies at the very centre of Western culture, we will draw on one of the most extreme products of this culture: fascism in Germany between the wars. The fascist perception of the body, male and female, does in fact aid us to understand the anxieties and desires expressed in the form of the two antagonistic machines. A parallel 
comparison, this time diegetic, between this film and The Terminator (Cameron, 1984), the first episode in the series, will help us to evaluate how these representations influence the narrative content of Terminator 2.

Terminator 2 (Cameron, 1992), aussi bien dans les thèmes qu'il aborde que dans sa facture, est un puissant témoignage de la fascination ressentie par la culture occidentale, américaine en particulier, envers la technologie. Le film, descendant déjà lointain de Star Wars (Lucas, 1977), utilise des effets spéciaux hautement sophistiqués pour représenter des machines imaginaires dont la sophistication est telle qu'elles peuvent ignorer les lois de la physique. Le spectateur est donc confronté à une image doublement stupéfiante qui semble bien souvent éluder tout aspect narratif par son simple impact visuel. Il est alors facile de croire que ce sont surtout ces images, par leur aspect spectaculaire, qui poussent des millions de personnes à voir un tel film.

Mais Terminator 2, comme son ancêtre et comme tout autre film hollywoodien, raconte avant tout une histoire, dont les situations et les personnages renvoient à la culture du public. La diégèse, loin d'être aussi vide de sens qu'on pourrait le penser, garde ici toute son importance. Certaines images du film sont peut-être totalement nouvelles, elles n'en représentent pas moins des figures archétypales communes à l'histoire entière du cinéma américain. Ainsi, la forme, le rôle et le comportement des deux machines antagonistes sont dictés par un ensemble de codes culturels dont la signification, notamment sur le plan sexuel, ne saurait être considérée comme fortuite. Ces figures seront l'objet central de notre étude. Leur comparaison avec celles utilisées dans le premier épisode servira à désigner les principales questions soulevées par le film.

The Terminator (Cameron, 1984), récupérant une vieille tradition du cinéma américain, montrait une humanité victime de machines devenues folles. L'intrigue en était simple: un "cyborg '", incarné par Arnold Schwarzenegger, venu d'un futur cauchemardesque, cherche à tuer Sarah Connor, la jeune femme qui donnera naissance au sauveur de l'humanité. Le seul espoir de celle-ci est un homme, Reese, lui aussi venu du futur, mais pour la 


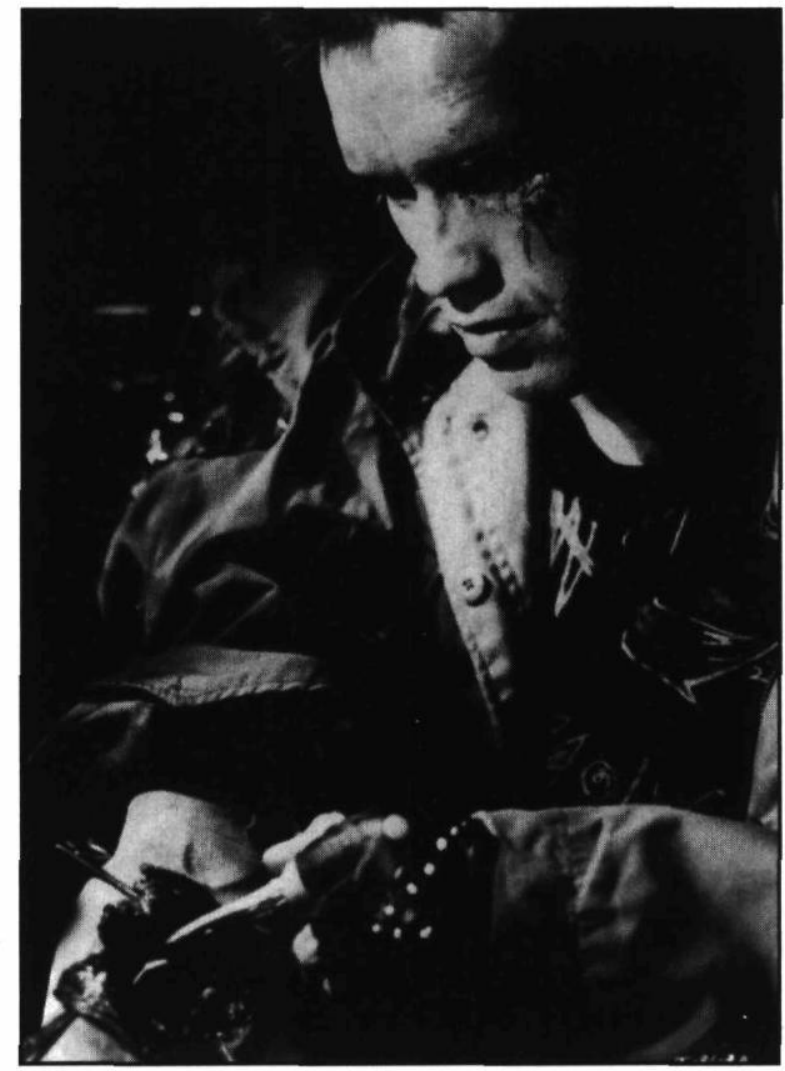

\section{Terminator 2 de James Cameron (1992)}

Collection Cinémathèque québécoise

protéger. Bien entendu, Sarah et Reese finissent par former un couple et parviennent ensemble à détruire la mauvaise machine.

Terminator 2 est apparemment la reproduction presque parfaite de ce scénario. Le film décrit l'affrontement de deux machines, à nouveau envoyées du futur. La première, appelée le "T-1000" et incarnée par Robert Patrick, cherche à tuer John Connor, le fils de Sarah. L'autre, interprétée par Arnold Schwarzenegger, essaie de les protéger, lui et sa mère. Les trois protagonistes ont aussi pour mission de faire disparaître les restes du robot détruit dans The Terminator, afin que la compagnie Cyberdyne ne puisse jamais mettre au point Skynet, l'ordinateur qui déclenchera l'holocauste nucléaire. 
On le voit, peu de changements apparents. Cependant, quelques différences cruciales nous poussent à considérer le deuxième épisode non comme le remake du premier, mais bien comme sa relecture.

La plus importante différence est la transformation du personnage négatif de Schwarzenegger en héros sauveur de l'humanité. Machine meurtrière, il est devenu le nouveau père du jeune John Connor et passe son temps à lui sauver la vie. Les créateurs du film ont donc jugé bon de réutiliser le cyborg. La célébrité de l'acteur est bien sûr à prendre en compte, mais malgré cette célébrité, il doit revenir à l'écran dans la peau du même personnage. Plus important encore est le fait qu'il meurt à la fin du film et ce, non en défendant John et Sarah, mais après les avoir sauvés, en se suicidant.

D'autre part, tous les effets spéciaux du film sont utilisés pour représenter les deux machines antagonistes, mais ils le font de manières fort différentes. Si Schwarzenegger n'a pratiquement pas changé, il a face à lui un nouvel ennemi qui va le mettre à mal bien plus férocement que dans tous ses autres films. Et avec ce nouvel ennemi doivent venir les justifications de sa destruction, qui prendront leur source aussi bien dans son rôle que dans son apparence.

Autre différence importante: Terminator 2, empruntant un chemin pour le moins inhabituel à Hollywood, est absolument dépourvu de toute intrigue amoureuse. Ingrédient quasi obligé du succès commercial, cet élément a été volontairement (?) laissé de côté et son absence signale que la puissance émotionnelle du film, essentielle à l'adhésion du spectateur, prend sa source ailleurs.

Ces trois particularités nous permettent de mieux définir notre approche du film. Celle-ci sera basée à la fois sur l'utilisation qu'il fait des codes narratifs hollywoodiens et sur la manière dont il choisit de représenter le corps de ses deux machines. Plus précisément, nous allons montrer comment une analyse des représentations du corps masculin permet d'expliquer les modifications narratives apportées par Terminator 2. C'est à travers ces deux angles de lecture que nous parviendrons à comprendre comment le film parle des rapports entre technologie et identité sexuelle dans la culture américaine. 
Que ce soit dans la littérature ou au cinéma, le rôle de la machine a toujours été dicté par le sexe qui lui était attribué. Le personnage du robot Maria dans Metropolis (Lang, 1928), instigateur de la révolte qui plongera la ville dans le chaos, est l'archétype de la femme fatale mythique. À l'inverse, Hal, le robot de 2001, A Space Odyssey (Kubrick, 1968), dont la volonté de contrôle le pousse à tuer l'équipage du navire, est indiscutablement masculin. De même, les androïdes de Blade Runner (Scott, 1982) se comportent de manières absolument opposées suivant leur sexe: les femmes sont impulsives et irrationnelles alors que les hommes restent toujours froids et maitres d'euxmêmes. Si l'on garde à l'esprit le fait que l'immense majorité des films sont écrits et produits par et pour des hommes, il apparaît clairement que ces représentations sexuées de la technologie reproduisent parfaitement les conceptions occidentales, donc masculines, de l'identité sexuelle. La féminité y est toujours dangereuse parce que mystérieuse et incontrôlable. La masculinité peut parfois devenir menaçante lorsqu'elle veut imposer un ordre trop rigide à la société. Mais qu'en est-il de Terminator 2 ?

J'ai parlé plus haut de la forme des deux machines comme étant le premier indice de leur véritable signification. Le choix d'Arnold Schwarzenegger a une importance capitale dans l'élaboration de son personnage. Le corps de l'acteur, élu cinq fois de suite "Monsieur Univers", possède une présence et un impact qui permettent facilement de le faire passer pour une machine. Sa carrure et la force qu'elle laisse supposer contribuent à donner une impression d'invincibilité et de puissance. Cette impression est renforcée par l'origine même de cette apparence: l'acteur est le produit ultime de la culture du body-building. C'est de là que vient sa célébrité, pas de son "jeu d'acteur" (Robert de Niro) ou de son "charme" (Mickey Rourke), mais de son physique incroyablement artificiel. Son corps est le résultat d'un travail scientifique qui a permis un développement rationnel et contrôlé de chacune de ses parties. Les machines qui l'ont créé l'ont façonné à leur image et on vient le voir pour cela.

Face à lui, le corps de Robert Patrick semble chétif. Beaucoup plus petit, très mince, il semble par comparaison sans substance. Même différence dans leur visage: celui de Schwarzenegger est 
large et très masculin alors que celui de Patrick est fin, presque féminin. Plus que toute autre caractéristique, c'est le degré de masculinité qui différencie, qui oppose les deux personnages.

Cette opposition est parfaitement reconduite par le filmage des deux antagonistes. Le personnage de Schwarzenegger garde toujours un statut actif dans l'image. Même au début du film, alors qu'il entre pourtant nu dans un bar, il se comporte immédiatement d'une manière si violente que nous n'avons pas le temps de nous arrêter à la contemplation de son physique. La fascination exercée par le film sur le spectateur ne vient pas d'une beauté esthétisée, mais de ses actions. Le reste du film va dans ce sens: il ne s'offre pas à notre vision, mais la dirige, comme il domine et dirige entièrement la narration.

Le T-1000 n'a pas du tout la même fonction en tant qu'image. L'analyse féministe a bien montré comment, dans le cinéma classique, l'apparition de la star féminine a pour effet d'interrompre la narration, le temps que la caméra nous fasse découvrir son corps. Or, les multiples transformations de ce personnage ont elles aussi pour effet d'arrêter l'action pour nous offrir le spectacle, quasi hypnotique, de ses formes. De plus, la caméra ne s'identifie jamais à lui, elle le place au contraire comme élément irréductiblement extérieur au spectateur ${ }^{2}$. En devenant ainsi l'objet passif de notre regard, il se positionne exactement comme la femme-objet du cinéma américain, ce qui continue de l'opposer à Schwarzenegger. Le physique de Robert Patrick et la plastique langoureuse de ce corps semi-liquide contribuent d'ailleurs pleinement à cette passivité dans l'image.

Leurs corps diégétiques, imaginaires renforcent cette première observation. L'utilisation des effets spéciaux est à ce titre remarquable. Le film utilise principalement la technologie dont nous avons parlé pour représenter les capacités étonnantes des deux personnages, pour montrer ce qui les rend spectaculairement supérieurs. Or, on retrouve ici le même axe d'opposition.

Commençons par la technologie extra-diégétique. Si Robert Patrick est bien un acteur réel, sor alter ego numérique est totalement virtuel. Il n'existe "matériellement" que dans une succession binaire du "zéro" et du "un", conservée dans la mémoire d'un ordinateur. La technique de représentation du 
T-1000 est donc abstraite, post-technologique en elle-même. Arnold Schwarzenegger, la star la plus célèbre et la mieux payée d'Hollywood, pour les raisons que nous venons d'expliquer, donne au contraire une matérialité totale à son personnage. Produit d'une technologie bien concrète, celle du body-building, son corps est un effet spécial en lui-même dont la force vient de son caractère profondément immédiat, matériel.

Examinons maintenant la technologie diégétique des personnages. Celui de Schwarzenegger est caractérisé par une incroyable résistance, une quasi-imperméabilité aux agressions extérieures. La séquence où des policiers tirent sur lui sans parvenir à arrêter sa progression est celle qui met en avant de la façon la plus claire cette caractéristique: il avance sans crainte, en fait sans montrer aucune émotion, alors que les balles ricochent sur son corps sans même le ralentir ${ }^{3}$. Même lorsque son enveloppe de chair est percée, elle révèle un intérieur de métal où l'on peut discerner les pièces solides qui constituent son squelette indestructible. La source de sa puissance est bien dans cette invulnérabilité : c'est elle qui, grâce aux effets spéciaux, est constamment mise en avant par le film. Sa psychologie suit le même principe. Rien ne lui échappe, il semble ne ressentir aucune douleur ou émotion et reste toujours entièrement maître de lui. C'est une sorte d'armure animée.

Son adversaire, dans la représentation duquel sont concentrés les effets spéciaux les plus étonnants du film, possède les caractéristiques inverses. Cette machine post-technologique a la faculté d'adopter n'importe quelle forme en modelant son corps de "métal liquide" à volonté. Ses multiples transformations révèlent une masse informe et sans consistance qui coule d'un endroit à l'autre pour se déplacer. Elle n'offre absolument aucune résistance aux attaques extérieures, au contraire, elle les absorbe ou les laisse la traverser. Ainsi, nous pouvons voir à plusieurs reprises les balles faire éclater sa chair chromée, la percer de part en part ou déchirer toute une partie de son corps si bien que nous pouvons voir à travers les trous ainsi formés. Malgré cette perméabilité totale, elle est parfois projetée en arrière par le choc des balles, mais les impacts se résorbent dans un bruissement spongieux et elle se relève. 
Ce personnage possède certes la même impassibilité que l'autre, mais ce manque d'émotion est ici lié à des caractéristiques totalement différentes. Le T-1000 est une menace pernicieuse, qui se déguise, se travestit, se cache, s'infiltre pour frapper en traitre. Sa froideur n'est pas une preuve de force. Dans le discours du film, elle est source de crainte plutôt que d'admiration, car elle révèle une cruauté calculée et lâche. Sa gestuelle exprime aussi quelque chose de mécanique, mais qui n’a rien à voir avec la puissance rigide de son adversaire: ses vêtements toujours impeccables, son regard fixe, ses mouvements extrêmement mesurés et précis ainsi que sa froide assurance lui donnent plutôt un air de maniaque sexuel. Cette tension extérieure laisse supposer un intérieur différent, tout juste contenu et caché, mais prêt à surgir pour agresser ses victimes.

Or, le film nous donne plusieurs fois l'occasion d'assister à ces agressions. Contrairement au cyborg, le T-1000 n'utilise pratiquement pas d'arme à feu. Il approche ses victimes, toujours des hommes, et transforme son propre fluide intérieur en lame métallique qu'il utilise pour percer leur corps, toujours au niveau de l'abdomen ou de la tête.

En plus d'être liquide et protéiforme, le T-1000 s'acharne donc à mutiler des hommes en pénétrant leur corps dans une promiscuité mise en avant par le film. Si l'on se remémore le fait qu'il poursuit un jeune garçon dans le but de lui infliger le même sort, il apparaît clairement que ce personnage est surtout dangereux parce qu'il possède tous les traits attribués par la culture occidentale à l'homosexualité. Cette caractéristique lui permet de réunir deux forces opposées. Nouvelle machine, nouvelle image, il est aussi nouveau personnage puisqu'il incarne à la fois l'ordre dominateur de Skynet et une sexualité ambiguë, donc tout aussi dangereuse.

Ses agressions sont le danger qui menace l'intégrité physique de Schwarzenegger, source, nous l'avons vu, de sa puissance hypermasculine. L'homme positif ${ }^{4}$, représenté ici selon les critères occidentaux de la masculinité idéale, doit triompher d'un ennemi dont l'hostilité est intimement liée à une identité sexuelle équivoque.

Cette première constatation doit maintenant nous amener à une compréhension plus profonde du film. Résumons la nature 
des forces en présence. D'un côté, un homme-machine dont la première caractéristique est l'invulnérabilité physique et psychologique. De l'autre, une entité au contraire totalement perméable, qui absorbe et envahit le corps des hommes. Il doit être possible d'aller au-delà de cette approche descriptive. Quelles conclusions pouvons-nous tirer de ces observations? Comment nous renseignent-elles sur l'objet de notre étude, le rapport entre technologie et identité sexuelle? Et surtout, comment expliquent-elles les différences narratives entre les deux films?

Ces observations prennent toute leur importance lorsqu'on les étudie à l'aide des travaux du chercheur allemand Klaus Theweleit. Son livre, Male Fantasies, qui analyse les écrits produits par les anciens membres des Freikorps allemands, est une réflexion globale sur la notion occidentale de masculinité qui éclaire puissamment un film tel que celui-ci ${ }^{5}$. Pour des raisons d'espace, nous n'expliquerons pas les fondements de sa théorie, son analyse descriptive suffisant à illustrer notre propos.

Theweleit montre que les soldats fascistes ont une perception déformée de la réalité. Cette perception est basée sur un rapport ambivalent, de fascination et de peur, envers tout ce que notre culture attribue à la féminité. Cette notion recouvre tout ce qui est liquide, indifférencié, mouvant et changeant. De façon particulièrement intéressante, elle englobe aussi ce que Theweleit appelle "l'intérieur" du soldat, ce qui coule en lui : aussi bien les liquides corporels que les émotions, le plaisir ou les pulsions. Plus intéressant encore, il est possible de rassembler toutes ces catégories sous le concept de désir. Ces flots extérieurs et intérieurs mettent continuellement la personnalité du soldat en danger d'éclatement et/ ou de dissolution. Pour s'en protéger, le soldat fasciste doit se constituer, par la discipline et l'entraînement physique notamment, un "corps-armure". Cette armure sert à réprimer ses flots intérieurs, en fait à empêcher que quiconque et surtout le soldat lui-même puisse penser qu'ils existent. Elle agit comme une structure rigide qui soutient le sujet, lui permet d'exister, de se mouvoir, d'interagir avec le réel. Isolé par une telle interface, il peut supporter le contact avec ses semblables. Bien sûr, il ne peut s'agir de rapprochements physiques ou émotionnels, car toute sensation de plaisir crée une brèche dans 
l'armure par où peut s'écouler la faiblesse féminine des fluides intérieurs. Le corps du soldat devient ainsi une forteresse assiégée par des forces liquides, liquéfiantes, qui doit rester hermétiquement close, tendue, rigide pour pouvoir continuer à exister. À l'intérieur de cette enveloppe impénétrable, les constantes frustrations (principalement sexuelles) qu'il doit s'imposer accumulent une énergie qui augmente encore la tension continuelle qu'il éprouve.

Une conséquence immédiate de cette perception du corps est un rapport particulier aux armes à feu. Seules ces armes sont en effet capables de révéler l'intérieur de l'adversaire en détruisant ses frontières corporelles, sans que l'homme prenne le risque de le toucher. Cette décharge violente d'énergie est virtuellement la seule que le soldat puisse se permettre.

Un point extrêmement important dans l'argumentation du livre est le soin avec lequel Theweleit démontre que ces perceptions ne sont qu'un aspect extrême de notre conception conventionnelle de la "féminité". Ici encore, il nous est impossible de rendre compte de l'analyse historique proposée par l'auteur. Mais il est clair que l'étude de l'histoire des femmes et des rapports entre les sexes, menée maintenant depuis plus de deux décennies, soutient parfaitement cette hypothèse.

Revenons à notre objet d'étude. Après la description que nous avons faite des deux machines antagonistes, l'importance d'une telle analyse devient évidente. Une telle proximité entre deux modes de représentation du corps masculin devrait nous aider à comprendre les enjeux posés par Terminator 2.

Cette hypothèse est pleinement confirmée par la description que nous avons faite du cyborg. Theweleit relève à maintes reprises la volonté, plus ou moins clairement exprimée, chéz le soldat fasciste de devenir une machine. Terminator 2 montre à quel point cette transformation va dans le sens d'une telle perception, de notre perception du corps.

Le squelette indestructible du cyborg est la promesse tangible parce que visuelle d'une ordonnance parfaite de notre intérieur si mystérieux et donc terrifiant. L'ensemble de ses caractéristiques est un écho parfait des conceptions fascistes du corps. Le physique de Schwarzenegger, totalement discipliné par des 
machines, est la meilleure représentation possible de cette nouvelle masculinité. La machine, mécanique pour le corps, électronique pour l'esprit ${ }^{6}$, promet de clarifier absolument le désordre interne, l'incertitude identifiée à la féminité, qui met tant en danger l'existence de la masculinité. Comme chaque individu a sa place précise dans la société fasciste, le corps mécanisé est composé de pièces autonomes, qui interagissent sans jamais se mélanger ni quitter leur position ou leur rôle initial. Ainsi organisée, la chair ne menace plus de manifester sa faiblesse féminine sous la forme de sang, de souffrance, de plaisir ou d'émotions. L'homme peut alors exister sans avoir à redouter de voir cette faiblesse dissoudre les fragiles frontières de son identité.

Face à ce corps, la représentation du T-1000 prend elle aussi une autre signification. Interprétée selon les travaux de Theweleit, elle se révèle hostile parce qu'elle est l'antithèse absolue de Schwarzenegger. Pas seulement de son personnage, mais de l'acteur lui-même, comme nous l'avons vu. Une telle créature aurait jailli des pires cauchemars des soldats fascistes. Sans forme définie et donc sans limites, menaçant toujours de déborder, elle est l'incarnation visuelle de tout ce qui aurait pu leur faire horreur. Ses caractéristiques physiques sont celles attribuées à la féminité menaçante. Au lieu d'être contenue par une enveloppe rigide, sa chair est immédiatement accessible et ô combien visible, toujours présente et prête à entrer en contact avec les autres corps.

Sa faculté d'absorber les attaques masculines est particulièrement révélatrice de cette nature féminoöde. L'arme et la balle étant les seules extensions tolérables du corps de l'homme, le fait pour lui de les voir disparaître dans une telle masse informe sans pour autant l'affecter constitue un spectacle angoissant. En effet, la décharge d'énergie ne s'opère pas véritablement puisque l'armure adverse n'est pas percée et, plus terrible encore, puisque avec la balle est absorbée une partie de l'homme lui-même. Ceci est particulièrement visible dans une des séquences finales du film. Il s'agit ici d'un échange de coups, mais l'exemple n'en est que plus probant. Ne parvenant pas à garder son adversaire à distance, le cyborg subit ses attaques corporelles répétées et faiblit inexorablement à ce contact. Dans un dernier effort, il 
frappe le T-1000 au visage, mais son poing ne fait que le traverser. Celui-ci emprisonne alors son bras dans une gangue métallique et fait couler sa tête de côté pour pouvoir continuer à lutter. Au cours de cette séquence, le cyborg est paralysé par ce qui se passe et ne peut rien faire pour se libérer. Il est littéralement vidé de toute énergie puisque celle-ci s'est perdue dans la masse informe de son adversaire. Ce contact aux résonances manifestement sexuelles a dissous l'homme, a épuisé sa capacité à défendre son identité face à une force qui n'est qu'un gouffre indifférencié engloutissant tout ce qui le touche.

Lorsqu'il parvient à s'approcher de sa victime, nous avons vu que le T-1000 perce son corps avec sa propre chair. Cette autre métaphore sexuelle a un résultat symbolique semblable. En violant ainsi les frontières des hommes, le T-1000 ne fait pas que les castrer. $S$ 'arrêter à cette première interprétation systématisante empêche de percevoir toutes les implications de nos observations. Cette union place les deux corps dans une promiscuité qui efface les différences rigides nécessaires à la survie de l'identité masculine définie selon les termes énoncés plus haut. Ultimement, le T-1000 est dangereux, car il est l'image de la faiblesse interne du corps de l'homme et qu'il menace à chaque instant de le mélanger, de le faire retourner à celle-ci.

Arrêtons-nous un instant. Le but de cette étude n'est pas de déterminer si Terminator 2 ou Robocop sont des films "fascistes" ni de savoir si leurs auteurs ou leur public souffrent de troubles de la personnalité. Une telle approche emprisonnerait l'analyse dans des jugements de valeur extrêmement réducteurs. Contrairement à la très grande majorité des travaux sur le fascisme ${ }^{7}$, Male Fantasies ne considère pas son objet comme un Autre "anormal" et "condamnable" dont il se dissocierait prudemment. Ni comme un passé révolu avec lequel nous, observateurs "rationnels" de l'histoire, n'avons plus rien à voir. Bien au contraire. Les soldats des Freikorps ne sont pas des monstres inhumains motivés par leur ignorance ou leur cruauté, ce sont avant tout des hommes. C'est un point de rencontre entre leurs textes et notre objet d'analyse dont l'importance est fondamentale. Mais ce lien, cette proximité pour le moins dérangeante, ne doivent pas nous pousser à les rejeter comme de mauvais objets que 
l'on observe à distance. Pour comprendre le fonctionnement de Terminator 2, il faut avoir ressenti l'excitation et le plaisir qu'il procure. Ces sentiments prouvent à quel point ce film, malgré (à cause de) son imagerie positivement fasciste, nous touche et nous parle de notre perception de la réalité.

Il s'agit ici de comprendre comment ces films, loin de donner une vision "fasciste" du monde, révèlent à quel point une telle vision est constitutive du rapport entre masculinité et technologie dans la culture occidentale et américaine en particulier. Terminator 2 montre que plus ce rapport devient étroit, plus sa représentation exige des images que l'on retrouve dans le discours fasciste. N'oublions pas la donnée la plus importante concernant les films hollywoodiens: comme les romans écrits par les soldats fascistes, ils sont faits par des hommes et pour des hommes. Ils ne laissent pas parler les femmes, mais les montrent, les mettent en scène, à travers le filtre déformant d'une certaine acception de la "féminité". L'intérêt principal du livre de Klaus Theweleit se trouve dans cette constatation : ces soldats représentent simplement l'aspect pathologique de la perception occidentale du corps masculin. Hollywood fonctionne sur les mêmes bases qu'eux, mais s'arrête, heureusement, au stade de la représentation.

J'ai parlé plus haut de l'importance capitale des armes à feu dans ces films. Sauf lorsqu'il est attaqué par le T-1000, qu'il doit le plus souvent affronter corps à corps, le personnage de Schwarzenegger en fait constamment usage. Le filmage insiste alors sur deux étapes de l'action: nous voyons le cyborg pointer son arme avec un geste continu, contrôlé et précis. Il tire et nous avons presque toujours le temps de voir une flamme, et son bras absorbant le recul. Le plan suivant montre invariablement l'impact de la balle faisant éclater la chair et projetant la victime en arrière. Précisons immédiatement que cette représentation est commune à tout le cinéma hollywoodien. Comme la conception occidentale de la féminité est caricaturée par le fascisme, Terminator 2 ne fait que caricaturer la relation homme-arme du cinéma américain.

Cette description correspond parfaitement à la fonction assignée par les soldats fascistes à leurs armes, ainsi qu'à l'image 
mentale qu'ils en avaient. Par fusion avec elle, l'homme adopte sa rigidité, le métal protège son existence. Elle lui permet ensuite de décharger son agressivité sur sa cible sans avoir de contact physique avec elle. Le soin apporté par le film à la représentation des effets de cette décharge est révélateur: blessures, explosions, vitres, objets, voitures, immeubles fracassés, tout tend à montrer qu'une quantité énorme d'énergie se libère. L'intensité du plaisir et de l'excitation ressentis par le spectateur au cours de ces séquences sont d'ailleurs révélatrices de cette fonction libératrice.

Les balles ont aussi l'avantage de projeter la cible loin du tireur, empêchant toute possibilité de contact physique; elles font le vide autour de l'homme. Ceci est particulièrement évident lorsque les protagonistes tirent sur le T-1000. Bien que réalisant l'inefficacité de leurs armes, ils continuent à les employer pour le repousser. Le filmage insiste alors sur l'efficacité apparente de cette stratégie pour ensuite montrer comment elle échoue de plus en plus totalement. Le suspens est construit sur le risque permanent de proximité avec cet Autre, proximité qui, nous le savons, est mortelle ${ }^{8}$.

Nous avons vu, au début de ce travail, comment une brève comparaison permet de montrer à quel point l'articulation différente d'éléments identiques fait prendre aux films deux directions opposées. Notre analyse explique ces modifications narratives en leur attribuant des causes et un sens précis. Il est maintenant possible de comprendre ces changements puisque nous en connaissons la source: la conception "fasciste", en fait occidentale, du corps masculin.

Dans le premier épisode, les deux protagonistes, Sarah et Reese, étaient humains. Leur force, ce qui les rendait positifs dans le discours du film, se situait dans l'humanité faillible de leur relation qui les opposait à la froideur génocidaire des machines. La chair, représentée principalement par la séquence où Reese et Sarah font l'amour, était alors le seul espoir de l'humanité.

Margaret Goscilo a montré comment The Terminator parvient à faire passer Sarah sous la domination masculine de Reese ${ }^{9}$. Celle-ci accepte de devenir la mère du sauveur de 
l'humanité et de suivre la route désignée par son compagnon. Dans Terminator 2, nous la retrouvons, lavée des traces de la maternité, sous l'apparence dictée par ce changement. Elle est athlétique, ses muscles sont saillants et sa silhouette robuste. Elle passe d'ailleurs de longues heures à s'entraîner dans sa cellule. Dure et impitoyable, elle est aussi experte dans les arts martiaux que dans le maniement des armes. Son sens aigu de la discipline lui fait même reprocher à son fils d'être venu la secourir. Comme tout film hollywoodien, c'est-à-dire masculin ${ }^{10}$, Terminator 2 est placé devant deux alternatives dans son traitement de la féminité: objectifier ou masculiniser. Deux décennies de féminisme rendent la première délicate. La seconde semble par contre satisfaire les lobbies et le public. Bien sûr, dans le cadre d'un film aux représentations aussi extrêmes, la femme acceptable (contrôlée) est celle qui aura perdu toutes les marques que notre culture attribue à la féminité.

Ces changements narratifs, inextricablement liés aux choix de représentation des corps, posent une nouvelle famille, solution à l'écroulement catastrophique du premier épisode. Dans The Terminator, Sarah vivait seule. Même si le film s'acharnait à prouver qu'elle n'était pas faite pour cela ", il donnait tout de même une vision assez libérée de la femme, notamment sur le plan sexuel ${ }^{12}$. De plus, la fin du film voyait la mort de Reese et laissait Sarah seule face au futur. Terminator 2 reconstitue la famille sous un père parfait parce que invulnérable, secondé par une femmehomme soumise. Elle et le jeune garçon, dont l'œdipe semble avoir été effacé par un père aussi exceptionnel, sont tous deux prêts à utiliser toute la puissance de la masculinité rigide pour sauver l'humanité.

Parallèlement à la transformation de Sarah, en faisant du cyborg son héros diégétique, Terminator 2 modifie radicalement les données de l'affrontement. L'enjeu du film n'est plus la survie de la chair, auparavant garante de la pérennité de l'humanité, mais de la masculinité rigide, manifestement la seule force capable d'accomplir cette tâche. La féminité, au sens conventionnel du terme, acceptée comme vitale dans le premier épisode, a été complètement expulsée des personnages positifs et concentrée dans la (non-)forme terrifiante du T-1000. Le Terminator n'est 
pas devenu amical et positif par la fantaisie des scénaristes, il l'est devenu parce qu'il était déjà la vedette du premier film. Et ceci parce que, dans notre culture, son corps, idéalement masculin (i.e. impénétrable) est la garantie absolument sûre de l'identification émotionnelle du spectateur.

Ceci nous amène à expliquer la principale différence narrative entre les deux films. Le premier épisode avait besoin d'une intrigue amoureuse, aussi bien diégétiquement que pour respecter les représentations et les codes narratifs hollywoodiens. Ce film n'en a pas besoin parce qu'il ne parle que du danger que de tels contacts recèlent. La sexualité ne peut plus apparaitre que par le contact cauchemardesque du T-1000 puisque cette sexualité fait partie de ce que l'homme a rejeté, visuellement et narrativement, pour atteindre un niveau d'invulnérabilité supérieur. Mais Terminator 2 ne quitte en rien le chemin tracé par son prédécesseur et l'ensemble de l'histoire occidentale, les soldats de Theweleit le prouvent. Il ne fait qu'approfondir des représentations qui ont toujours existé.

Le suicide du héros n'a pas d'autre sens. Avant de descendre dans le métal en fusion, celui-ci explique à John qu'il ne peut pas pleurer. L'homme mécanisé n'a plus à retenir ses larmes, il n'en a pas. Émotions, sentiments, chaleur humaine lui sont aussi étrangers que le corps de la femme, car il a dû les abandonner eux aussi en endossant son armure. La douleur et la mort ne lui font pas peur: il ne vit pas. Ce sont les femmes qui vivent, lui ne fait qu'exister. En tombant dans la fosse, le T-1000 se dissout lentement en hurlant, il se débat et s'accroche à la vie en reprenant les visages des personnes qu'il a imitées: il meurt. Le cyborg y descend volontairement et reste rigide jusqu'au bout, il ne fait que disparaître. Son poing dressé lève le pouce à l'adresse du jeune garçon, preuve ultime de son insensibilité qui devient alors le lien rapprochant les deux hommes.

Nous sommes à présent en mesure de préciser la nature de la relation qui existe, dans le cinéma américain, entre technologie et identité sexuelle. L'analyse combinée de la représentation du corps humain et de l'utilisation des codes narratifs révèle à quel point la narration hollywoodienne dépend des données culturelles liées à l'identité sexuelle. Dans le cas de Terminator 2, elle 
permet de comprendre comment la représentation de la technologie est influencée par ces données et construit deux machines, deux technologies, bien différentes.

La première, utilisée par l'homme individualiste, est entièrement positive. Et ce, car elle est l'homme individualiste. En accord avec ses valeurs, elle n'est ni compliquée ni abstraite. Après tout, il n'a besoin que d'une armure et d'armes à feu. Le squelette métallique du cyborg est un écho de la naïveté des jouets guerriers de Star Wars. Cette naïveté, cet infantilisme devrions-nous dire, montre bien que ces machines sont uniquement le produit d'une vision déformante de la réalité, d'une représentation. La technologie (masculine) hollywoodienne, intra et extra-diégétique, n'a qu'un but: créer une réalité où l'homme est invulnérable.

Élément très important: il est désigné comme dépassé par la post-technologie du T-1000. Mais son triomphe, comme celui de tant de héros américains, prouvera que la technologie masculine traditionnelle, individualiste et libérée de la féminité, permet d'échapper à la corruption d'une modernité devenue folle (aux deux sens du terme, malheureusement). Mais pour régner à nouveau, cette masculinité a besoin d'un nouveau corps, construit par des machines et fonctionnant (i.e. existant) comme une machine. Ne nous y trompons pas, le spectateur sait pertinemment quel acteur interprète ce rôle. Ce que nous voyons est autant un "cyborg" que Schwarzenegger, nouvelle incarnation de la masculinité triomphante. Seul cet acteur peut donner une représentation visuelle satisfaisante d'une telle masculinité.

L'autre machine est totalement hostile sur les deux plans d'analyse. Image d'une féminité terrifiante, sa modernité maladive est aussi bien représentée par son homosexualité, qui devient ici symbole de la mort de l'humanité, que par son hypersophistication. Narrativement, elle a été créé par Skynet, l'ordinateur qui a conclu que les humains voulaient lui nuire et qui a tenté de les exterminer. Résultat de l'excès de rationalisation et d'organisation de la société (ici rien de moins que l'informatisation du système de défense américain), le T-1000 n'est certes que technologie, mais technologie machinique, c'est-à-dire inhumaine. Et ici, comme dans tout le cinéma hollywoodien, 
" inhumain" veut, bien sûr, dire anti-individualiste et (donc) antimasculin, ce qui rend à coup sûr cette altérité irréductible et légitime entièrement sa destruction.

Qu'en est-il des femmes? Il est facile de percevoir des personnages tels que Sarah ou Ripley (trilogie Alien: 1978, 1986, 1992) comme les reflets de changements qui ont récemment bouleversé la société nord-américaine. Dans une telle lecture, ces personnages de femmes fortes représentent les femmes réelles qui ont rejeté le rôle secondaire que notre culture leur a toujours assigné pour prendre une place à part entière dans la société.

Malheureusement, un tel point de vue ignore la véritable nature du cinéma américain, Terminator 2 en est une excellente preuve. Ce film, comme tous les autres, ne donne qu'une vision extrêmement déformée de la réalité, vision rendue encore plus hystérique par les tentatives de prise de pouvoir des femmes. Linda Hamilton et Sigourney Weaver sont des femmes, leurs alter ego sont des personnages. L'apparence, le rôle et les actions de ces personnages montrent clairement que le cinéma hollywoodien n'a absolument pas modifié sa perception et sa représentation des femmes. Celles-ci sont toujours faibles, mystérieuses et extérieures puisque pour prendre une place centrale et active $^{13}$ dans la narration, elles doivent devenir des hommes: invulnérables et non désirantes. Seuls leur corps et leur attitude masculinisés leur donnent le droit de se défendre toutes seules ou de porter des armes ${ }^{14}$. Hollywood sait changer d'emballage pour pouvoir continuer à vendre. Ce travail, qui cherche à comprendre les représentations du rapport homme-machine, a dû revenir aux rapports homme-femme. Ce retour obligé est la preuve que nos notions culturelles définissant ce rapport sont absolument centrales au processus de représentation hollywoodien. Naturellement, ces notions étant basées sur la même perception des corps masculin et féminin que celle qu'avaient les soldats fascistes, il ne faut pas s'étonner que les films hollywoodiens produisent des représentations semblables. Naturellement, tant que ces notions n'auront pas véritablement changé, ce cinéma produira toujours le même discours. 


\section{NOTES}

1 J'emploie le terme "cyborg" (cybernetic organism) à dessein. Ce mot, issu de la culture populaire, désigne un être constitué à la fois de parties mécaniques et organiques. Ce n'est donc pas un "robot", mais un homme-machine, ce qui a une certaine importance pour la suite de ce travail.

2 Alors que dès la première séquence où Schwarzenegger apparaît, le film nous donne le plaisir de percevoir la réalité à travers son regard électronique. Le fait que ce procédé était déjà employé par le premier épisode révèle encore une fois l'importance de ce personnage et de son traitement.

3 On retrouve exactement la même scène dans Robocop (Verhoeven, 1987), dont le personnage principal est un descendant direct du Terminator de James Cameron.

4 Pas simplement positif parce qu'invulnérable. Sarah estime en effet qu'il aurait fait un bien meilleur père pour John que tous les hommes qu'elle a rencontrés. De plus, tous les hommes du film sont présentés comme déficients, par faiblesse ou par aveuglement devant le danger qui menace l'humanité. Seul le cyborg, qui amène du futur une connaissance universelle, voit et sait ce qu'il faut faire.

5 Lauteur utilise principalement les travaux de Deleuze et Guattari, notamment le cycle Capitalisme et Schizophrénie (Paris: Minuit, 1972-1980), ainsi que les travaux de Melanie Klein et Margareth Mahler.

6 Voir à ce titre Total Recall (Verhoeven, 1990), dont le héros, interprété par Schwarzenegger, voit ses capacités physiques décuplées après avoir acquis électroniquement la mémoire d'une autre personne.

7 Et contrairement à la majorité des travaux historiques en général. Le passé, notre passé, n'est pas un ailleurs temporel coupé du présent. Nous en sommes le produit, c'est un lieu commun dont il ne faut pas négliger l'importance. Pour le comprendre, il faut le vivre à travers les textes, en percevant et en analysant nos propres réactions face à eux.

8 Le film Aliens (Cameron, 1985) est un autre exemple, encore plus caricatural, de cette utilisation des armes. Perdus dans le labyrinthe sombre et humide construit par les créatures meurtrières, les soldats paniquent et tirent dans toutes les directions. Mais malgré cette tentative désespérée de sauvegarder leur identité, ils sont presque tous happés un à un par cette obscurité mortelle dont l'origine est, faut-il le préciser, hystériquement féminine. L'analogie entre cette séquence et les scènes de combat des films sur la guerre du Viêt-nam ouvre des pistes dont ce travail n'est que l'amorce.

9 Voir "Deconstructing The Terminator", Film Criticism, vol. 12, n 2 (19871988), p. 37-52. Article à mon avis très important par la précision avec laquelle il démonte le prétendu féminisme du film.

10 "Masculin" car conçu, écrit, produit et réalisé par des hommes. Mais aussi et surtout parce que fait pour une société dont les hommes restent le centre et les femmes, le "continent obscur». Il ne s'agit évidemment pas ici d'opposer des caractères biologiques (masculin/féminin), mais de préciser que nous percevons les valeurs, différentes et inégales, que notre culture leur accorde.

11 En insistant sur sa maladresse dans son travail de serveuse et surtout en concentrant les caractéristiques de la mauvaise féminité (sexualisée et antimaternelle) dans son amie et colocataire Ginger (que le Terminator tue sauvagement, cela va sans dire). Voir "Deconstructing The Terminator", Film Criticism, vol 12, n 2 (1987-1988), p. 37-52.

12 L'expérience sexuelle de Sarah est en effet positive, car elle lui permet d'aller vers Reese, que ses souvenirs guerriers semblent avoir jusqu’à présent éloigné (protégé?) de toute sexualité. 
13 Sharon Stone est un personnage central de Basic Instinct (Verhoeven, 1991), mais avec un corps tel que le sien (un corps incroyablement désirant), on ne s'étonne pas qu'elle soit mystérieuse, dangereuse et bisexuelle. Pour faire une vraie héroïne, rien de tel qu'un crâne rasé et des épaules bien carrées.

14 Ou de se suicider pour sauver l'humanité, comme le fait Ripley, en bonne disciple de Schwarzenegger, à la fin d'Alien 3 (Fincher, 1992), emportant avec elle le fruit terrifiant de ses entrailles.

\section{OUVRAGE CITÉ}

Theweleit, Klaus. Male Fantasies, vol. 1-2. Minneapolis: Polity Press, 1987-1989. 